\title{
Incidence and pattern of implant fractures: A long-term follow-up multicenter study
}

\section{CLINICAL RESEARCH - \\ PERI-IMPLANT BIOLOGY}

\author{
Jae-Hong Lee \\ Department of Periodontology, Daejeon Dental Hospital, \\ Wonkwang University, College of Dentistry, Daejeon, Republic of Korea
}

\section{Abstract}

Among a total of 19087 implants in 8501 patients (7838 male and 663 female) placed over 9 years, fractures were observed in 70 implants (0.4\%) in 57 patients (50 male and 7 female). Cases with less than $50 \%$ bone loss had a higher incidence of horizontal and vertical implant fractures limited to the crest module, which are defined as Type I fractures $(n=13,18.6 \%)$. In contrast, cases with $\geq 50 \%$ severe bone loss exhibited a higher incidence of Type II vertical fractures $(n=22,31.4 \%)$, extending beyond the crestal portion ( $P=0.001)$. Type III fractures $(n=5,7.1 \%)$, defined as a horizontal fracture beyond the crestal module, were also observed.

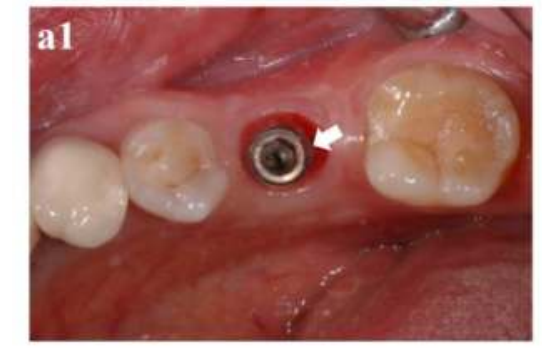

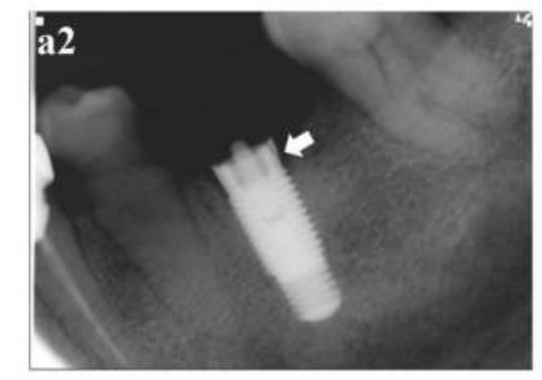

Type I

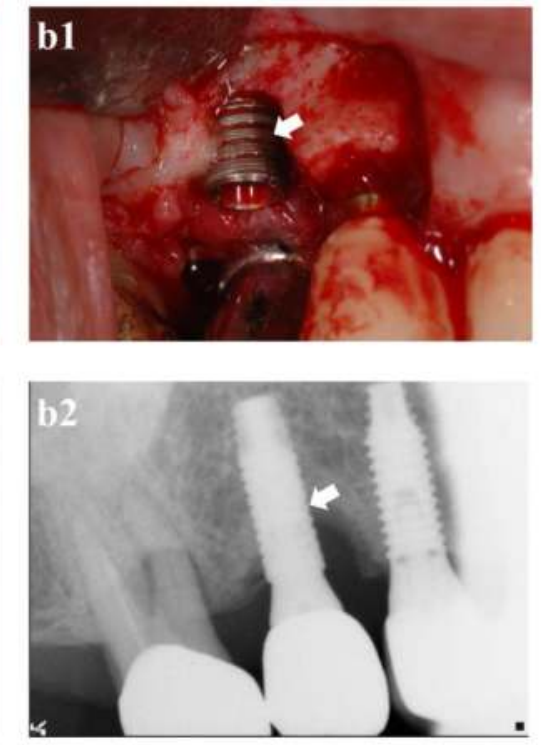

Type II
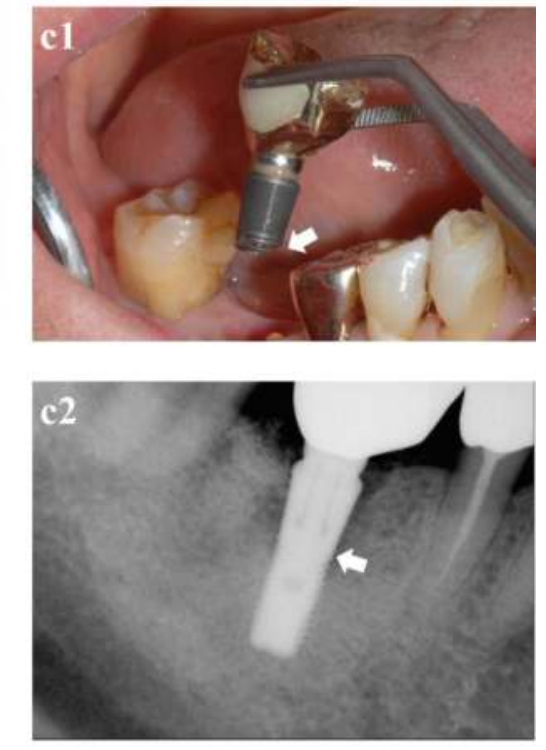

Type III

\section{Background and Aim}

Currently, there is incomplete understanding of the fracture patterns in the implant and their association with clinical factors. The aim of this study was to investigate the incidence and pattern of implant fracture by using 9-year, long-term multicenter follow-up data.

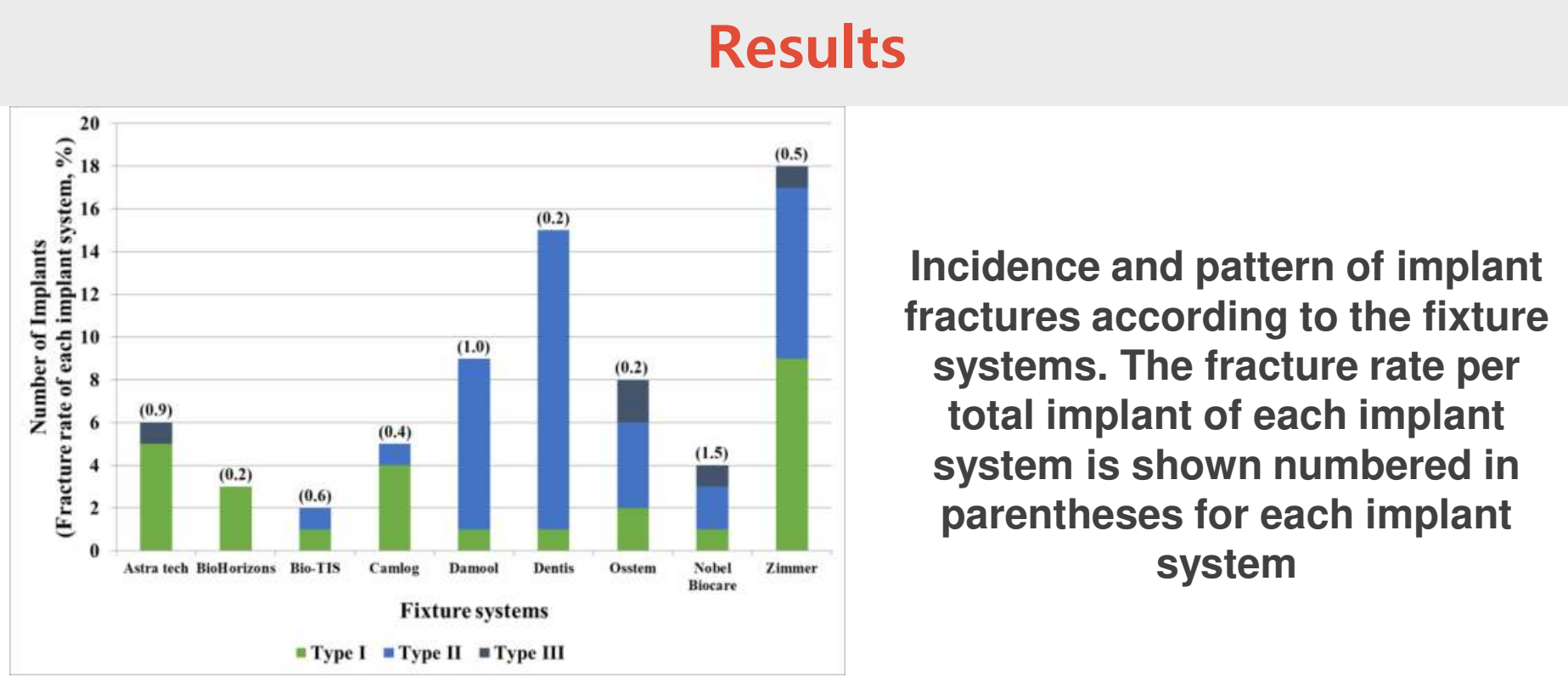

20

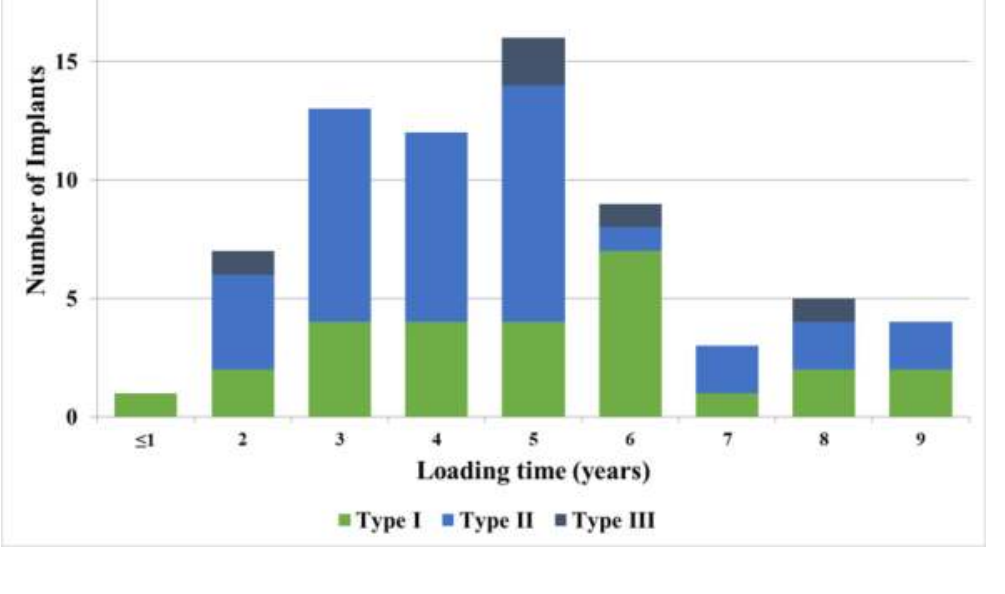

Incidence and pattern of implant fractures according to the duration of functional loading

Incidence and pattern of implant systems. The fracture rate per (otant system is shown numbered in system

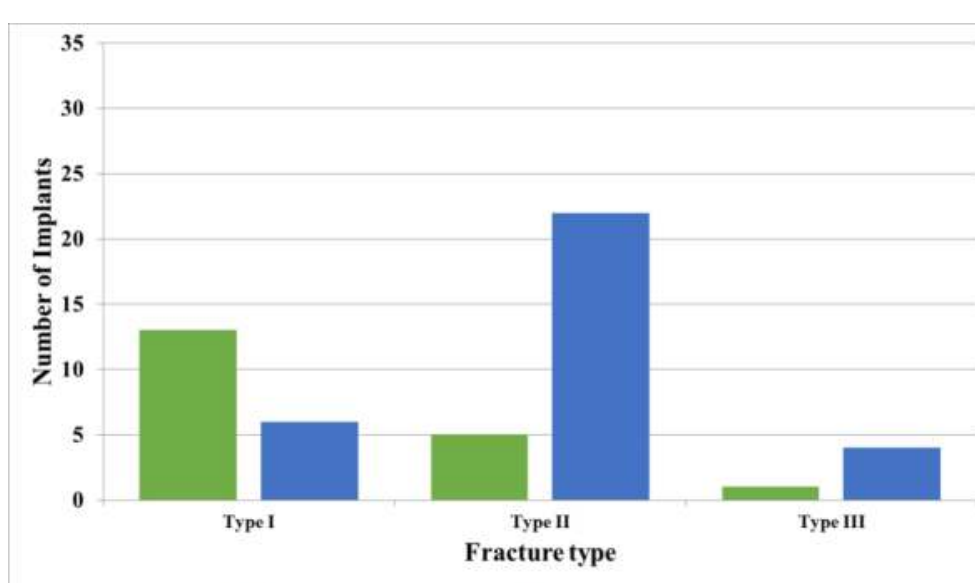

Incidence and pattern of implant fractures according to periimplantitis-induced marginal and vertical bone loss

\section{Conclusion}

Peri-implantitis-induced marginal and vertical bone loss and manufacturing-induced defects were considered to be major factors in implant fracture. Therefore, using clinically verified implant systems and striving to minimize bone loss by preventing and actively treating peri-implantitis is essential to reduce implant fractures.

\section{Methods and Materials}

\section{References}

The association of the incidence and differences in fracture patterns with clinical factors (based on patient variables and implant variables) was assessed for statistical significance using the Chi-square and Fisher exact test, as appropriate.
Funding information: Basic Science Research Program through the National Research Foundation of Korea (NRF) funded by the Ministry of Science, ICT \& Future Planning, Grant/Award Number: NRF-2017R1C1B5014849 